FORMATION Formation emploi

Revue française de sciences sociales

101 | janvier-mars 2008

Numéro anniversaire : Regards croisés sur les

relations formation-emploi

\title{
La recherche de liens entre la formation et l'emploi : une institution et sa revue - Un point de vue -
}

Searching for links between training and employment: an institution and its journal in retrospect

Berufsbildung und Beschäftigung: Ein Forschungsinstitut und seine Zeitschrift La búsqueda de vínculos entre la formación y el empleo: una institución y su revista. Un punto de vista

Lucie Tanguy

\section{CpenEdition} Journals

Édition électronique

URL : http://journals.openedition.org/formationemploi/57

DOI : $10.4000 /$ formationemploi.57

ISSN : 2107-0946

Éditeur

La Documentation française

Édition imprimée

Date de publication : 1 janvier 2008

Pagination : 23-40

ISSN : 0759-6340

Référence électronique

Lucie Tanguy, « La recherche de liens entre la formation et l'emploi : une institution et sa revue - Un point de vue - », Formation emploi [En ligne], 101 | janvier-mars 2008, mis en ligne le 31 mars 2010, consulté le 30 octobre 2020. URL : http://journals.openedition.org/formationemploi/57 ; DOI : https:// doi.org/10.4000/formationemploi.57

(c) Tous droits réservés 


\title{
Numéro anniversaire
}

\section{La recherche de liens entre la formation et l'emploi : une institution et sa revue - Un point de vuel -}

Lucie Tanguy*

\author{
Les discours sur la formation tout au long de la vie rappellent \\ ceux tenus sur l'éducation des adultes avant la loi de 1971. \\ Si on préconisait alors des dispositifs institutionnalisés répondant \\ à des demandes collectives, les politiques actuelles sont orientées vers la demande \\ et la responsabilité individuelle des apprenants.
}

La réflexion qui suit répond à une commande : mon point de vue sur l'évolution de la relation formation/ emploi. Je reviendrai sur cet énoncé pour rappeler, ce que j'ai déjà eu l'occasion de dire à maintes reprises, les attendus implicites qu'il autorise et les occultations qu'il contient. Tout d'abord, si j'ai accepté de me plier à cet exercice d'acrobatie intellectuelle sans disposer du temps nécessaire à une réflexion rigou-

${ }^{1}$ Ce texte n'aurait pu être réalisé dans le temps imparti sans l'aide de : Marie Baudry de Vaux (CEREQ) qui m'a fourni tous les documents et informations utilisés notamment (mais pas seulement) dans le premier paragraphe ; Daniel Blondet (DGESCO - Direction générale de l'enseignement scolaire) et Claude Sauvageot (DEPP - Direction de l'évaluation, de la prospective et de la performance) qui ont attiré mon attention sur l'importance de certaines questions ; Annick Kieffer (Centre Habwachs, ENS - École normale supérieure), Fabienne Maillard (DGESCO) et Françoise Ropé (université d'Amiens) qui, par leur lecture critique d'une première version de ce texte, m'ont permis de l'améliorer. Je les remercie tous très vivement. reusement argumentée, c'est que mon itinéraire scientifique s'est déroulé parallèlement à celui du CEREQ (Centre d'études et de recherches sur les qualifications) avec des croisements répétés et prolongés par une participation au comité de rédaction de la revue, depuis sa création en 1983 jusqu'en 1989 d'abord, par une coopération très féconde lors d'une mission officielle commandée par le Secrétariat

* Lucie Tanguy est sociologue, directrice de recherche émérite au CNRS, rattachée à l'Unité mixte de recherches GTM (Genre, Travail et Mobilités) - université Paris 10. Parmi ses dernières publications, deux ouvrages : Les Instituts du travail. La formation syndicale à l'université de 1955 à nos jours, PUR, 2006 et, en collaboration, Former pour réformer. Retour sur la formation permanente 1945-2004, 2007. 
d'État à l'Enseignement technique et professionnel sur les problèmes posés à la formation professionnelle des ouvriers et des employés par la politique d'élévation à $80 \%$ d'une classe d'âge au niveau du baccalauréat (Tanguy, 1991) ensuite, et enfin par la participation au conseil scientifique, de 2001 à 2005.

La teneur de mes réflexions tient pour une part à ce compagnonnage fait de rencontres et de disputes toujours fructueuses mais aussi à mon insertion dans la sociologie de l'éducation d'abord et dans la sociologie du travail ensuite, deux branches d'une discipline académique où cet objet n'occupe qu'une faible place $^{2}$, malgré des impulsions réitérées provenant d'individus ou d'institutions diverses au cours des trente dernières années. La perspective d'analyse adoptée ici provient également des différents collectifs de chercheurs que j'ai pu animer, depuis l'Introuvable relation formation-emploi. Un état des recherches en France, en 1985 jusqu'au dernier ouvrage de ce style, Former pour réformer. Retour sur la formation permanente 1945-2004 (Brucy et alii, 2007). J'ai souvent privilégié cette forme de recherche parce qu'elle me semblait être plus apte à cultiver des terrains encore trop désertés, à mettre en perspective des travaux de parcelles isolées et, ce faisant, à établir un corpus d'énoncés susceptibles d'être mis à l'épreuve et de guider les jeunes chercheurs débutants à s'affranchir de ce goût pour la nouveauté, que j'estime extrêmement préjudiciable au travail scientifique dans les sciences sociales. La propension à découvrir du nouveau n'est souvent qu'ignorance du passé où un même phénomène était désigné par d'autres mots.

L'esquisse d'analyse de la question posée « la relation formation/emploi », dont la définition même fait problème, ne peut évidemment être que dessinée à grands traits ${ }^{3}$ et à partir d'un point de vue que j'ai construit au fil des recherches menées.

Tout d'abord, je reviendrai sur la création du CEREQ en 1970 et sur les missions qui lui étaient attribuées, non pas pour montrer comment cette institution s'en

\footnotetext{
${ }^{2}$ Il est, par exemple, ignoré dans cet ouvrage, qui entend faire une synthèse de la recherche en sociologie du travail : Pillon Thierry, Vatin François, 2003, Traité de sociologie du travail, Toulouse, Octarès.

${ }^{3}$ C'est pourquoi les références bibliographiques se limitent à des travaux collectifs émanant, pour la plupart, du CEREQ lui-même.
}

est acquittée mais plutôt pour pointer quelques glissements entre les questions jugées centrales dans ces années et celles considérées comme telles durant cette dernière décennie. Je proposerai également d'examiner comment est née la revue pour occuper un espace autour de deux pôles désignés par la juxtaposition de deux notions, et entreprendre d'étudier l'ensemble des relations qui les lient l'un à l'autre. Je tenterai de montrer que le projet initial s'est ainsi trouvé réduit à une sorte de substrat appelé « la relation formation-emploi » qui appauvrit singulièrement l'ambition d'origine et a contribué à générer de mauvaises controverses sur des vrais problèmes.

Je tenterai ensuite de rendre compte de l'évolution de ce domaine de questions, à partir d'un ensemble de travaux antérieurs, en distinguant un peu arbitrairement deux périodes caractérisées par quelques traits majeurs. La première me paraît aller jusqu'aux années 80 dans la mesure où elle peut, a posteriori, être qualifiée comme une période de profondes transformations économiques et sociales où la formation a joué un rôle de levier important. La seconde, qui concerne les quinze dernières années, n'est pas moins marquée par des changements d'ampleur équivalente mais dont le sens s'est inversé. La formation est toujours convoquée comme instrument nécessaire à l'accomplissement de ces transformations dans l'emploi et le travail, mais, présentée comme un droit individuel, elle s'avère plutôt être une obligation pour garder un emploi. Les deux configurations distinguées se différencient surtout par une inversion des changements de politiques qui abandonnent toute référence à des changements structurels et laissent accréditer l'idée d'individus responsables de leurs situations.

\section{UNE INSTITUTION D'EXPERTISE PUBLIQUE}

Le CEREQ, créé en 1970, est officiellement défini comme un institut interministériel de recherche spécialisé au sein de l'Office national des informations sur les enseignements et les professions (ONISEP). C'est dire qu'il naît sur un terrain déjà occupé et sous une forme juridique qui mérite d'être soulignée : un statut interministériel caractéristique 
des réformes administratives impulsées dans le cadre des $4^{\mathrm{e}}$ et $5^{\mathrm{e}}$ Plans afin de mobiliser les forces économiques et sociales plutôt que d'administrer d'une manière réglementaire (Casella, 1999) ${ }^{4}$. Sa date de naissance elle-même appelle une analyse contextuelle pour lui donner sens. Elle se rapporte, comme il a été souvent signalé, à la planification et, singulièrement, aux travaux de la commission de la maind'œuvre, présidée par Jean Fourastié, durant les $\mathrm{IV}^{\mathrm{e}}$ et Ve Plans. «Le CEREQ est né d'un paradoxe et demeure dans une posture paradoxale», jolie formule de Ph. Méhaut (2001) pour dire que les missions de cet institut sont contenues dans ce postulat d'une adéquation de la formation à l'emploi, source d'un développement économique et social équilibré. Cette focalisation sur la formation comme facteur de modernisation économique et sociale a pu se réaliser dans le cadre de cette action publique de l'État qu'est le Plan, parce qu'elle avait déjà cours dans les grandes entreprises, dans les politiques régionales de reconversion industrielles, etc. $\mathrm{La}$ projection d'une économie plus productive exigeant une élévation générale de la qualification, au moyen d'une élévation du niveau de formation, a ainsi été présentée comme une définition rationnelle de l'intérêt général. L'héritage de l'ère planificatrice conservé par le CEREQ réside dans ce raisonnement d'experts de la prévision mais surtout, à mes yeux, dans cette pratique qui consiste à traduire des réalités multiformes en grandeurs mesurables.

L'année de naissance du CEREQ suit également l'explosion des luttes étudiantes entrainnant une critique radicale de l'Université, ainsi que celle du système éducatif en général. Elle précède de quelques mois l'édiction de la loi de 1971. La chronologie de ces événements est rarement mentionnée, bien qu'elle ait révélé la profondeur d'une crise sociale qu'il fallait juguler de toute part, comme le dit F. Ceyrac (entretien cité par G. Brucy 2007, p. 253), alors Président du CNPF (centre national du patronat français), «... 1968, c'était l'ébranlement, il fallait éviter à tout prix que ça recommence. Avec la formation, on pouvait conduire le changement par l'adaptation du personnel ».

${ }^{4}$ Philippe Casella a montré que l'élaboration d'une politique de formation des adultes est une expression de ce mouvement de réformes administratives.
Le programme originel du CEREQ, tel qu'il est présenté par sa direction ${ }^{5}$ est ambitieux, tant par le domaine des questions qu'il entend traiter que par la complexité des analyses qu'il prétend mener. Les limites de son domaine d'études sont mal définies. Elles se déplacent et s'étendent corrélativement à la manière dont est abordée l'étude des relations entre deux sphères d'activités sociales, jusqu'alors séparées et désignées par deux termes, formation et emploi, le plus souvent conjuguées au pluriel durant la décennie 1970. La connaissance d'un tel domaine exige, est-il dit, de combiner la macroéconomie avec les biographies professionnelles, les études de contenu d'emplois avec celles des formations ellesmêmes. Si les contours de ce domaine sont indéterminées, son centre est explicitement désigné : la qualification, qui vient d'être définie dans le Plan comme une résultante du niveau de formation. L'analyse de la qualification est effectivement, durant cette première décennie, constamment affirmée être «l'objectif central du CEREQ,... qui correspond à la fois à sa vocation la plus originale et à l'apport le plus constructif qu'il puisse offrir au pédagogue » (CEREQ, 1973). Cette tâche implique, est-il précisé, d'établir un mode de connaissance " des acquisitions de compétences, de les mesurer et de les valider, recherche qui doit être poursuivie dans les systèmes de formation par les équipes spécialisées en sciences de l'éducation ». Un peu plus tard, en 1976 (CEREQ, 1976), le CEREQ est désigné comme "un institut national d'analyses et de prévisions qui a pour fonction essentielle d'étudier l'adaptation des formations et des méthodes d'enseignement aux transformations du monde du travail ». Pour réaliser un tel programme, la direction du CEREQ entreprend de développer des liens avec le milieu universitaire et, singulièrement, avec les sciences de l'éducation (nouvellement instituées dans les universités). Une mission pédagogique est créée en son sein qui, "en relation avec les conseillers pédagogiques, aura pour tâche de transposer les résultats de l'analyse du travail en objectifs de formation précis permettant d'actualiser les

${ }^{5}$ Note d'information $\mathrm{n}^{\circ} 1$, «Premières orientations méthodolo-
giques des travaux du CEREQ » (sans date), directeur de la publi-
cation G. Ducray qui est aussi le premier directeur du CEREQ de 1971 à 1981 . 
enseignements existants, du BEP (brevet d'études professionnelles) aux diplômes d'ingénieurs ». Par la suite, le CEREQ se détachera des sciences de l'éducation pour se rapprocher d'une manière préférentielle des économistes.

L'évocation des premiers pas du CEREQ, faite ici, laisse voir un certain nombre de processus qui avaient cours durant ces années et qu'il a contribué à cristalliser: la définition de la qualification par la formation et celle de la formation par les compétences acquises. À cette date, la notion de qualification fait l'objet de controverses scientifiques entre sociologues et entre économistes du travail qui opposent deux conceptions: l'une dite «substantialiste» parce qu'elle retient un facteur unique, la formation, pour rendre compte de la hiérarchie du travail, l'autre relativiste et conflictuelle parce qu'elle n'est abordée qu'au travers des enjeux, luttes et processus sociaux qui concourent à sa codification dans les classifications, par exemple, et à son évaluation en termes de salaires. Les études du CEREQ se situent clairement dans la première perspective, plus propice à une action directe sur l'offre de formation, mais surtout parce qu'elle correspond au point de vue adopté par la direction de cette institution dans le débat, «...il n'est pas correct de considérer que toute élévation de la qualification entraîne nécessairement un glissement des grilles de classification et provoque de ce fait des charges salariales plus lourdes. Une politique de qualification et de meilleures utilisations de la main-d'œuvre implique que les gains de rendement et d'efficacité technologique soient explicitement pris en compte » (CEREQ, 1978).

La formation, elle-même, est réduite à son caractère instrumental : l'acquisition de compétences requises pour l'exercice du travail. Cette représentation, épurée de toute référence à la formation sociale et civique, préfigure déjà le modèle pédagogique des compétences mis en place dans les années 90 . Présente en termes logiques, cette conception ne se réalise, plus tard, qu'avec les outils de mise en correspondance des diplômes et des emplois que sont les référentiels.

Ce retour sur les discours, les programmes, les énoncés des positions du CEREQ, ou plutôt de sa direction, car comme toute institution elle n'est pas une entité monolithique, ne saurait omettre cette donnée contextuelle qui sous-tend la création de cet organisme d'étude ainsi que les missions qui lui sont attribuées : une croyance dans la maîtrise des phénomènes économiques et sociaux par la science. Le raisonnement qui sous-tend les orientations des études menées dans les années 70 relève d'une pensée de style positiviste où la mesure est considérée comme un gage de la scientificité. L'importance accordée aux outils pour observer l'évolution des capacités professionnelles, à partir des entrées dans la vie active (EVA), ou des «systèmes de travail » (Répertoire français des emplois), et pour agir sur les politiques d'emploi et de formation professionnelle, trouve sa justification dans cette posture d'esprit largement partagée par les élites étatiques de ces années 1960-1970. Loin de s'ériger en dogme, cette pensée s'incline devant les faits observés: la discordance entre les mouvements qu'on entend réguler d'une manière équilibrée. C'est ainsi qu'un rapport (CEREQ, 1978, p. 5) souligne «qu'il devient essentiel de procéder à un examen approfondi des critères de sélection à l'embauche des entreprises, ceux-ci n'étant pas exclusivement liés à des exigences de formation », puisque les cheminements professionnels laissent apparaître des déclassements professionnels et des statuts précaires. Le questionnement sur les liens entre la formation, le travail et l'emploi, qui vise toujours à résoudre les problèmes posés, s'inscrit alors dans l'observation d'ensembles beaucoup plus vastes : les entreprises et leurs organisations du travail, leurs politiques de recrutement, de gestion du personnel, les systèmes techniques, l'emploi. Les études sectorielles réalisées qui embrassent les relations entre ces différentes dimensions en constituent une illustration. Certaines demeurent des références, celles appliquées au bâtiment et à la chimie par exemple.

Toujours présent, l'emploi ne devient central qu'avec la montée du chômage et celui des jeunes notamment. La remarque d'A. d'Iribarne (directeur adjoint du Céreq de 1974 à 1981) sur l'infléchissement des modes d'analyse et le délaissement progressif du terrain des entreprises me paraît, à première vue, traduire un changement qui devrait être examiné de plus près. Je ne pense pas, contrairement à H. Bertrand (alors directeur du CEREQ), que les 
travaux menés plus récemment sous le label « compétences » puissent être considérés comme des investigations équivalentes. Ce retrait laisse un manque de connaissances qui n'est pas comblé par les études en provenance d'autres organismes : de fait, l'analyse du travail s'est singulièrement réduite (d'Iribarne, 2001 ; Bertrand, 2001).

\section{Une revue pour faire exister un champ en sciences sociales appliquées}

Lorsqu'elle paraît, en 1983, J.-F. Germe, adjoint au directeur et président du comité de rédaction, définit la ligne éditoriale de la revue par son objet et son style. Le premier est plutôt désigné en creux: «Formation Emploi n'est ni une revue sur la formation, ni une revue sur l'emploi. Mais Formation Emploi a l'ambition, en revanche, d'être une revue consacrée aux problèmes de la relation entre la formation et l'emploi et à l'évolution des qualifications. " Arrêtons-nous sur cet énoncé et sur le titre retenu qui élimine le travail pour lui préférer l'emploi, signe des problèmes existant de ce côté. Alors que le pluriel était d'usage durant la décennie précédente, le singulier s'impose dans un syntagme qui va connaître le succès que l'on sait dans les milieux professionnels spécialisés d'abord, puis dans l'opinion commune. La juxtaposition des deux termes dans le titre, qui laisse totalement ouvert l'espace interprétatif, est sans doute à l'origine de l'adoption généralisée de cette expression nominale, dont l'indétermination autorise une grande diversité d'expressions. Décideurs des politiques publiques, de l'emploi et de la formation, mais aussi parents d'élèves ou salariés peuvent y projeter leurs attentes respectives. Délestée de toute préposition, cette locution apparaît comme une relation ayant la force de l'évidence. Du pluriel qui reconnaissait la diversité des situations et liens, on passe au singulier, coup de force symbolique, qui généralise et érige « la relation formation/emploi » au rang de concept. J'ai pris la mesure de la force des mots, et de ceux-ci en particulier, lors de la réception de cet ouvrage collectif que j'ai dirigé, L'Introuvable relation formation emploi (1986) et qui reprenait cette locution. A posteriori, je me suis rendue compte que, par le type d'analyse qu'il faisait d'un questionnement social, cet ouvrage avait contribué à donner forme à des représentations, à des politiques qui cherchaient à établir des relations entre deux ordres de phénomènes, la formation et l'emploi, et à faire apparaître ces relations comme nécessaires alors que son propos était à l'opposé. En bref, par maints aspects, cet ouvrage a pu conforter une propension à parler de «la relation formation emploi » au singulier et à substantifier des phénomènes qui ne peuvent être pensés qu'en termes de processus. La majorité des études menées aujourd'hui dans ce domaine font apparaître la formation comme la condition première d'accès à l'emploi, en occultant les rapports sociaux qui président à cette relation entre titres et places.

Le style donné à la revue témoigne, à l'inverse, d'un attachement constant de l'institution à la diffusion d'enquêtes fondées sur des méthodes statistiques qui guident l'action des politiques, mais pas seulement. On ne saurait trop insister sur ce rôle de veille méthodologique que le CEREQ exerce sur la scène publique, évitant que le débat sur des questions vives ne dérive en polémique partisane. Tout comme l'institution entendait, dès ses origines, instaurer des relations étroites avec la recherche universitaire, la revue se propose elle aussi d'être le porte-parole du débat scientifique en ouvrant ses colonnes à des travaux menés dans des centres différents. Bien qu'en partie réalisée, cette intention se heurte à une double série d'obstacles : la nécessité de publier les résultats d'études des équipes qui travaillent directement en son sein ou dans les centres régionaux qui lui sont associés d'une part, et au faible nombre de travaux menés par des chercheurs universitaires d'autre part. Vus sous cet angle, les articles de cette revue se sont, pendant longtemps, relativement peu confrontés aux autres publications portant sur les mêmes questions, comme en témoignent les bibliographies qui leur sont assorties. C'est le revers d'une institution qui occupe de fait, et non de volonté, une position de quasi-monopole. ${ }^{6}$ Mais on assiste, depuis

\footnotetext{
${ }^{6}$ Le CEREQ compte 160 personnes, dont 125 à Marseille. Seize centres lui sont associés, qui emploient 35 personnes rémunérées par l'institution et 80 autres personnes qui contribuent à temps plein à son programme d'activité. Mais, comme le remarque souvent Annick Kieffer du Centre Maurice Halbwachs, le CEREQ a, plus tôt que l'INSEE, mis ses fichiers d'enquêtes à la disposition des chercheurs. Leur usage est limité par le nombre d'intéressés. J'ajouterai que, durant les années où je siégeais au comité de rédaction, aucune sorte de censure ne s'est manifestée sur les propositions d'articles reçues.
} 
quelques années, à une ouverture significative de la revue : par l'élargissement de son comité de rédaction à des chercheurs extérieurs et par l'accroissement du nombre de publications émanant de divers horizons. La parution, impulsée récemment, de numéros spéciaux sur des thématiques, objets de débats, tels que ceux consacrés à l'immigration, à l'Europe et, tout dernièrement, à de nouveaux courants de recherches tels que celui d'une économie des capacités traduit cette affirmation d'une revue spécialisée dans le champ des sciences sociales en France. Comme telle, elle constitue, aujourd'hui plus qu'hier, un instrument qui structure la recherche dans ce domaine?

\section{UN PUISSANT LEVIER DE TRANSFORMATIONS}

Je propose de dresser la toile de fond sur laquelle le CEREQ a été construit et qui éclaire certains aspects de son fonctionnement durant ses deux premières décennies d'existence. À première vue, cet examen peut ne pas être considéré comme très pertinent, parce que trop éloigné de la production du CEREQ. Il permet pourtant de situer celle-ci dans un mouvement de mise en place d'une nouvelle activité sociale qui, par les diverses propriétés qui lui ont été conférées, s'est imposée comme un instrument de changement ayant un intérêt général. La formation est, en effet, représentée comme un bien universel recherché aussi bien par l'État et les entreprises que par les salariés.

L'idée d'une formation tout au long de la vie est aujourd'hui adoptée dans tous les pays développés, comme le rappelle Kjell Rubenson, observateur spécialisé dans l'analyse des politiques éducatives sur la longue durée en Suède et dans la comparaison avec d'autres pays. Il formule ainsi un constat que chacun peut faire dans son propre pays : «On trouvera difficilement un document sur la politique

\footnotetext{
${ }^{7}$ Parallèlement, le CEREQ adopte une politique de publications d'ouvrages de synthèse rassemblant des études menées par ses chercheurs et ceux d'autres institutions voisines, tel que celui intitulé Des formations pour quels emplois ? (qui réunit vingt-deux contributions) sous la direction de Giret J.-F., Lopez A., Rose J., 2005.
}

éducative, l'économie ou la cohésion sociale qui ne renvoie pas, d'une manière ou d'une autre, au concept d'apprentissage tout au long de la vie. Considéré comme solution aux problèmes économiques et sociaux du monde industrialisé, ce mode d'apprentissage est devenu une espèce de "Nouvelle Jerusalem"... Ce point de vue a déplacé le débat auparavant centré sur les acquis cognitifs fondamentaux à la possibilité, pour les adultes, de s'engager dans un apprentissage tout au long de la vie.» (Rubenson, 2001)

En France, ce mot d'ordre nommé « Formation tout au long de la vie» se greffe sur la notion de formation, qui tend à prendre une place équivalente à celle d'éducation. Elle a été construite, en France, au cours des années 1950-1960, dans un contexte marqué par la recherche d'un accroissement de la productivité économique qui apparaît alors comme un impératif aux dirigeants politiques, aux dirigeants économiques mais aussi aux hauts fonctionnaires et à de nombreux intellectuels; contexte qui a donné naissance au CEREQ. Cet acte résultait d'un choix, parmi d'autres possibles, réalisé par des élites réformatrices, en vue de répondre à des problèmes singuliers.

Évoquer le chemin suivi par la Suède en ce domaine est, de ce point de vue, très instructif, dans la mesure où l'éducation des adultes y est plus développée et les inégalités moindres qu'ailleurs. Héritée d'une tradition remontant au $\mathrm{XIX}^{\mathrm{e}}$ siècle, cette situation provient très largement des mouvements d'éducation populaire. Les théories du capital humain ont été mises à mal dans ce pays qui conjuguait, jusqu'au début des années 60, "un niveau scolaire moyen proche de celui du Portugal avec un marché $d u$ travail comparable à celui de la RFA » (Rubenson, 2001). Dans la généalogie qu'il fait des réformes mises en place au cours de ces quatre dernières décennies, K. Rubenson souligne que, tout en programmant une forte croissance de la scolarisation, le ministère de l'Éducation, en accord avec les syndicats de salariés, a choisi d'intégrer l'éducation des adultes dans le système scolaire national afin de concilier efficacité économique et démocratie culturelle et politique. Une telle politique reposait non pas sur un principe abstrait d'égalité d'offre pour chacun mais sur la création d'une demande au sein des groupes sociaux peu enclins à suivre des études. Elle 
a été fortement infléchie tout au long des années 1980-2000, durant lesquelles les entreprises ont concurrencé les instances traditionnelles d'offre d'éducation que sont les municipalités et les associations. Il reste que ce pays est celui où le taux d'illettrisme est le plus bas de tous les pays de l'OCDE (Organisation de coopération et de développement économiques) et celui où la participation des femmes à la vie civique publique est la plus élevée : faits qui peuvent être attribués, selon Staffan Larsson (2001), à cette institution d'éducation populaire, caractéristique de la démocratie en Suède, "les cercles d'études » auxquels environ un million et demi de personnes participent chaque année.

De tradition solaire opposée, la France a connu des formes différentes d'appel à la formation en vue de moderniser l'économie ainsi que l'organisation et les relations sociales $\mathrm{du}$ travail. C'est d'abord au système scolaire que les politiques se sont appliquées pour produire les deux grandes «explosions scolaires » intervenues dans les années 50 d'abord et les années 80 ensuite. Elles se sont faites, on le sait, en termes d'élévation de niveau plus que de changements d'organisation et de contenus d'enseignement, si l'on excepte les filières professionnelles qui se sont profondément développées et renouvelées, des lycées jusqu'à l'enseignement supérieur. Je n'insisterai pas sur ces aspects qui sont maintenant connus de tous. Je soulignerai plus longuement ceux qui restent encore méconnus: l'instauration d'une formation permanente (ou continue, je néglige ici la variabilité des dénominations bien que celle-ci soit éminemment signifiante) qui s'est opérée, à l'initiative d'élites réformatrices œuvrant dans différents lieux de la société, pour finir par être codifiée dans une succession de lois. J'en donnerai deux exemples : l'action de la première génération des directeurs du personnel dans les grandes entreprises et celle des experts du Plan.

\section{L'adaptation des salariés au travail}

Les actions menées par la première génération des directeurs du personnel dans les grandes entreprises pour mettre en place des pratiques de formation, dès le début des années 50, étaient sous-tendues par un souci d'accroître la productivité mais aussi par la volonté de changer les relations de travail en rédui- sant l'autorité de la hiérarchie, en anticipant les conflits et en facilitant leur résolution par la concertation et la négociation. Les problèmes inhérents aux relations sociales ont ainsi été traduits en termes techniques permettant d'apporter des réponses qui faisaient accord sur le registre de la formation. Ces hommes de la formation se sont en effet présentés comme des techniciens - on dirait aujourd'hui des experts - artisans d'une politique contractuelle reposant sur le compromis et l'accord. Ils ont fondé et animé les associations, l'ANDCP (Association des directeurs et chefs du personnel), les GARF (Groupement amical des responsables de formation) et l'Institut Entreprise et Personnel, qui ont façonné ce domaine d'activités dans les entreprises. Ce sont les mêmes hommes qui ont fondé les premières institutions de formation au management en France.

Ils en donnent une définition qui, rompant avec les termes alors utilisés comme «perfectionnement, recyclage, adaptation, promotion », annonce celle aujourd'hui en usage. Elle est énoncée en termes de compétence, compétence qui est «la conjonction heureuse des connaissances, des aptitudes et de la bonne volonté " pour maintenir individuellement et collectivement l'ensemble du personnel adapté à l'activité de l'entreprise (Vatier, 1958). Définition qui évoque, on le voit, les principes des méthodes de gestion aujourd'hui en usage dans les entreprises, qui lient formation et compétences et qui explicitent la notion de compétences sur les trois registres du cognitif, de l'action et des comportements (en termes de savoir, savoir-faire et savoir être). La littérature consacrée à l'usage de cette notion dans l'École et l'entreprise et aux transformations qu'elle désigne est trop abondante pour citer les études les plus significatives ${ }^{8}$. De nature le plus souvent performative, plus rarement analytique et critique (Dupray et alii, 2003) ces études révèlent le penchant des sociologues à

\footnotetext{
${ }^{8}$ Nous avons nous-mêmes apporté une contribution à ces travaux avant l'inflation qu'ils ont connue dans la dernière décennie. Au risque de pêcher par immodestie, nous l'indiquons au lecteur parce qu'elle portait explicitement sur les outils techniques et les procédures élaborés pour la naturaliser. Lors de la publication de l'ouvrage cité, nous ignorions qu'elle était déjà introduite par les responsables de formation dans les grandes entreprises (Ropé et Tanguy, 1994).

${ }^{9}$ Cet ouvrage collectif, dans lequel divers spécialistes de sciences sociales examinent le traitement fait de cette notion par leurs disciplines est, de ce point de vue, une exception.
} 
s'emparer de prénotions, utilisées à des fins de résolution des problèmes sociaux, pour les présenter comme des notions expliquant des phénomènes qualifiés de «nouveaux » parce qu'ils n'étaient pas jusqu'alors immédiatement visibles sur la scène publique.

Dans les mêmes années, Jean-Léon Donnadieu (directeur du personnel chez BSN) crée un service intitulé « organisation-formation », promeut la notion d'organisation qualifiante (diffusée plus tard par les sociologues du travail) et avance une conception aujourd'hui acceptée, mais alors jugée irrecevable, selon laquelle la formation se définit par ses objectifs et non par ses contenus (Donnadieu, 1999). Par maints aspects, ces militants de la formation ont été les précurseurs de la pensée managériale qui s'est répandue deux décennies plus tard, en France.

\section{Qualification et productivité}

La représentation de la formation comme instrument de modernisation économique et politique a été généralisée, on l'a dit, dans cette action publique de l'État qu'est le Plan. Institué au lendemain de la Seconde Guerre mondiale, le Plan a été un lieu de rencontres, d'échanges et d'élaboration de cadres de perception communs entre ce qu'il est aujourd'hui convenu d'appeler les partenaires sociaux, qui avait pour ambition de transformer la société française à partir de son économie. Il a fait admettre sur la scène publique la nécessité de lier l'éducation à l'économie, promu la notion de formation, en lieu et place de l'éducation, l'a fait apparaître comme une grandeur mesurable et l'a posée au fondement de la qualification. Les cadres des politiques éducatives menées durant ces quatre dernières décennies trouvent là leurs origines: la modernisation économique suppose une élévation générale du niveau de scolarisation et l'établissement progressif du baccalauréat en norme.

Les artisans de cette représentation de la formation vecteur d'une économie compétitive et d'un progrès social qui lui est consubstantiel étaient des experts : en l'occurrence, les responsables de la commission de la main-d'œuvre dont le président, entre 1954 et 1970, est Jean Fourastié (ancien élève de l'École centrale, professeur au CNAM - Conservatoire national des arts et métiers -, enseignant la comptabilité à l'Institut des sciences politiques, auteur d'un ouvrage sur le retard de productivité pris par la France bien avant la guerre); Bertrand Schwartz (Ingénieur du corps des mines, ancien directeur de l'École des mines de Nancy, directeur du CUCES (Centre universitaire de coopération économique et sociale) est le président d'un groupe de travail chargé d'élaborer «la méthodologie»; Raymond Vatier (ingénieur, un des fondateurs de l'ANDCP et des GARF) est rapporteur d'un autre groupe de travail, créé au sein de la commission de la productivité. Ils ont élaboré une définition consensuelle de la formation qui l'a représentée comme un bien universel, qui est tout à la fois un moyen : de développer l'adaptabilité dans un cadre professionnel (dans l'intérêt des entreprises) ; de promotion et de mobilité professionnelle et individuelle (dans l'intérêt des salariés); et de dynamiser l'économie (dans l'intérêt national).

Apôtres du progrès économique et de l'élévation du niveau de formation ainsi défini, les experts du Plan projetaient qu'en 1970, $30 \%$ d'une classe d'âge atteindraient le niveau du baccalauréat alors que cette proportion était de $11,5 \%$ en 1960 . C'est ainsi que les principes directeurs des politiques éducatives mises en place dans la décennie 1980, et notamment celui érigeant le baccalauréat en norme, ont été institués. Bien que controversée, par les représentants du monde professionnel notamment, cette vision prospective, qui contenait en elle les déclassements professionnels aujourd'hui observés à grande échelle, s'est imposée au nom de la recherche d'un accroissement de productivité. ${ }^{10}$

\section{Un renversement de conception pédagogique}

Les pionniers de la formation pour adultes se sont tous employés, quels que soient leurs lieux d'action, à inventer des pédagogies qui ont pour caractéristique

\footnotetext{
${ }^{10}$ R. Poignant, rapporteur de la Commission Éducation au sein de ces mêmes Plans, fait valoir que ce déclassement relatif pour les individus sera tout bénéfice pour la société et l'économie, «à l'exemple de ce qui existe déjà aux États-Unis où l'absorption des diplômés dans l'économie s'effectue déjà sans difficulté mais dans des emplois qui ne requéraient pas, naguère, une qualification si élevée [...] Il est certain que cette "surqualification" de la maind'œuvre par rapport aux normes actuelles va devenir un facteur important de la "productivité économique". »"
} 
commune revendiquée de s'opposer aux pédagogies scolaires. Au sein de cet agglomérat d'actions très variées, Bertrand Schwartz apparaît comme l'un des fondateurs d'une doctrine de l'éducation permanente qui est devenue une référence commune au monde de la formation. Elle s'est élaborée dans la durée, mais les principes majeurs sont fixés dès les premières actions collectives menées, au début des années 60 , à l'occasion des grandes reconversions industrielles (des mines et de la sidérurgie) en Lorraine. Cette expérience $\mathrm{a}$, en effet, servi de creuset à l'élaboration d'une doctrine et à l'expérimentation de méthodes et de techniques pédagogiques : la formation a été conçue comme une action individualisée, liée aux parcours professionnels, qui se poursuit tout au long de la vie, s'organise en unités modulaires pouvant faire l'objet de validations réalisées en termes d'« être capable de ». Une théorie de la connaissance liée à l'action s'est ainsi diffusée et a influencé la pédagogie scolaire. On ne soulignera jamais assez combien les différents lieux où se mettaient en place des actions de formation continue ont été simultanément des lieux de réflexion et d'expérimentation pédagogiques qui ont donné naissance à des doctrines ensuite codifiées et étendues à la formation initiale, mais aussi, d'une manière indirecte, à tous les segments de l'appareil scolaire et à l'Université ellemême, comme on va le montrer un peu plus loin.

On voit ainsi que tous les pionniers de la formation ont été de véritables innovateurs et des réformateurs de la France des années 1950-1960. Ils ont plaidé pour des réformes à promouvoir en termes de changement des individus (et des salariés notamment) plus qu'en termes de changements des structures; l'obstacle à la réalisation des changements souhaités leur paraissant résider de ce côté. Les individus sont, en effet, presque toujours représentés par eux en termes de manques, sous l'angle de la privation des qualités nécessaires à l'accomplissement des changements souhaités : manque de connaissances, manque de capacités d'adaptation, manque de mobilité, manque d'ouverture d'esprit, d'attitudes, etc. Spontanément interprétée en termes cognitifs, cette perception du salarié en termes de manques qu'il faut combler s'est étendue aux représentations collectives, aux modes de vie, aux cultures de métiers, comme le montrent les politiques de reconversion industrielle. Cette focalisation sur les individus, représentés par leurs manques, dépossédés de leurs ressources effectives, est aujourd'hui exacerbée dans les discours et les politiques qui définissent la formation comme un co-investissement des entreprises et des individus pour acquérir et maintenir les compétences exigées par la recherche d'un accroissement de la productivité du travail. Les enquêtes portant sur l'insertion des jeunes ont pu, à l'insu de leurs auteurs, contribuer à diffuser cette représentation.

Des exceptions doivent être rappelées, parmi lesquelles B. Schwartz (1994) et M. David (1976), pour qui la formation doit avant tout être collective parce que les situations qui la requièrent résultent de déséquilibres et non d'insuffisances ou de manques de la part des individus.

Conçue à l'origine comme un puissant instrument de changement et un bien destiné en premier lieu aux plus démunis face à ces changements à faire advenir, la formation apparaît aujourd'hui sous une image inversée : un outil utilisé à des fins d'ajustement ou d'anticipation des mouvements de l'emploi et du travail. Loin d'être associée à la promotion projetée par les discours politiques des années 60 , elle participe activement à une intensification de la sélection opérée entre catégories d'actifs sur le marché du travail et dans l'exercice de l'emploi.

Nombre de faits, rappelés plus haut, sont à l'origine de cette expression nominale « la relation formation emploi », aujourd'hui assimilée à un nom commun désignant une chose établie. La catégorie de niveau de formation elle-même est maintenant utilisée comme une catégorie explicative, qui tend à se substituer à celle de catégorie socioprofessionnelle, en usage dans les années 1960-1980, pour rendre compte de la majorité des comportements sociaux et politiques.

Ces constructions sociales et symboliques, dont on mesure aujourd'hui les effets, reposaient sur le primat accordé à la productivité dont l'envers, le déclassement des diplômés ${ }^{11}$, était déjà pressenti. Elles enregistraient des pratiques en cours demandant aux salariés un effort de formation sans le

${ }^{11}$ Les analyses faites en ces termes contribuent, elles aussi, à naturaliser cette construction. 
reconnaître en termes de salaire et de place dans la division du travail.

Durant cette période, un changement radical d'orientation et de définition des politiques éducatives s'est opéré. Il a, on l'a vu, été introduit par les Commissions et le Commissariat général au Plan et non, comme antérieurement, par le ministère de l'Éducation nationale au regard des débats parlementaires et des actions provenant des agents du système éducatif lui-même (Briand et alii, 1993).

Les publications émanant de l'observation des entrées des jeunes sur le marché du travail à la sortie de l'école ont donné à voir les transformations réalisées au sein du système éducatif en réponses aux injonctions d'adaptation qui lui étaient faites. $\mathrm{Ce}$ faisant, le CEREQ coupait court aux polémiques récurrentes sur le caractère "inadapté» de ce système et accusant certaines organisations professionnelles d'employeurs d'être à l'origine du chômage des jeunes ${ }^{12}$. Les observations et les analyses menées l'ont été au moyen d'instruments construits à partir des questions sociales posées et des postulats qui les sous-tendent, confortant ainsi l'inflexion des politiques sans montrer ce qu'elle occulte: la minoration des fonctions premières de tout système éducatif, la socialisation de la jeunesse et l'élaboration de ce que le sociologue allemand Darhendorf (cité par Beate Krais (1995)) appelle « la rationalité cognitive d'une société moderne»; le gain en efficacité aux dépens de la démocratisation, laquelle impliquait, au moins un principe de recherche d'égalisation des chances; et enfin, l'absence, jusqu'à une date récente, d'une reconnaissance sociale sur le marché du travail (en termes de classification, de promotion et de salaire) des acquis cognitifs ainsi obtenus. Pourtant, et dans le même temps, l'expertise acquise par le CEREQ en matière de mise en relation de la sphère éducative avec celle de l'emploi s'est exercée d'une manière décisive dans la fabrication des diplômes, sous forme de veille technique tant au niveau de leur nombre que de leur périmètre professionnel. La réflexion menée par Fabienne Maillard (Maillard, 2005) sur cette activité

\footnotetext{
${ }^{12}$ Dont j'ai pu prendre une mesure lors de la réalisation de la mission évoquée précédemment et au sein du Haut comité ÉducationÉconomie-Emploi où je siégeais (début des années 2000).
}

mériterait d'être poursuivie dans ce sens. La masse des études réalisées au sein du CEREQ, à cette fin, représente, à mes yeux, une mine d'informations dont l'analyse montrerait précisément comment cette institution s'acquitte d'une des tâches qui lui ont été attribuées dès l'origine et qui est une des formes de mission d'expertise publique dont elle se revendique à juste titre.

\section{DROIT OU INJONCTION À LA FORMATION ET INDIVIDUALISATION}

La centralité accordée à la formation s'est accompagnée d'un ensemble de changements majeurs dont je mentionnerai ici les plus significatifs.

Le premier fait caractéristique de la dernière décennie, et souligné par tous les observateurs, concerne: la stagnation de l'élévation du niveau moyen de formation institué dans l'accès d'une classe d'âge au baccalauréat, qui voisine les $65 \%$, et celle de l'accès à l'enseignement supérieur, qui plafonne à $50 \%$ depuis 1995 ; la quasi-stabilité du nombre de jeunes qui sortent de l'école sans avoir les requis nécessaires, phénomène dont la loi de 1989 assurait l'éradication; et la consolidation de la hiérarchie produite, ou reproduite, par la formation continue qui creuse ainsi, en termes relatifs, les inégalités sociales.

\section{De l'éducation à la formation}

Tous les promoteurs de la formation partagent une croyance collective dans les vertus libératrices de l'éducation, mais la majorité d'entre eux se défient de l'Éducation nationale et critiquent l'« École» parce qu'elle reproduit les inégalités sociales. Cette critique s'est accompagnée d'un projet d'instauration d'une éducation des adultes libérée de l'autorité de l'État pour l'ancrer dans la société civile, voire dans un système éducatif englobant l'éducation des enfants et celle des adultes. Durant ces trente dernières années, un monde de la formation s'est non seulement développé en termes de dispositifs, de règles, d'acteurs, mais aussi de points de vue, de représentations qui ont 
imposé une conception instrumentale des actions éducatives dans leur ensemble.

Cette conception inspire très directement les politiques de ces dernières décennies qui ont placé les préoccupations de l'emploi au cœur du système éducatif, sur des modes différents et avec des intensités inégales selon les segments qui le composent. Le caractère général de cette référence est énoncé dans la loi d'orientation de $1989^{13}$ qui prescrit de conduire $100 \%$ d'une classe d'âge, au niveau V minimum. L'objectif ainsi attribué à l'appareil éducatif, non seulement opère un déplacement de l'ordre scolaire à l'ordre des qualifications, mais pose le niveau $\mathrm{V}$ (mesuré par les diplômes professionnels CAP et BEP - certificat d'aptitude professionnelle et brevet d'études professionnelles) comme minimum. La spécificité de la qualification ouvrière (désormais subsumée sous l'appellation « niveau $\mathrm{V} »$ ), s'est ainsi trouvée niée et présentée comme équivalente à une scolarisation nécessaire, à un moment donné, dans une société donnée. Formuler les politiques éducatives en termes de niveau de formation ou de qualification témoigne de cette volonté, toujours réaffirmée, d'établir des relations d'équivalence entre ces quatre registres différents de la réalité sociale que sont l'éducation, la formation, la qualification et l'emploi ; elle dénie la réalité du marché du travail et engendre illusions et désenchantement chez les jeunes notamment.

Cette croyance en la formation s'est quelque peu affaiblie ces dernières années, dans un contexte d'élévation généralisée d'une scolarisation prolongée. Le glissement progressif d'une gestion du personnel instituée en termes de qualifications négociées collectivement vers une gestion définie en termes de compétences validées au sein des entreprises peut être interprété comme une forme d'infléchissement des relations instituées au début des années 60 . Les débats qui ont cours aujourd'hui, et les pratiques qui les accompagnent (comme la loi sur la validation des acquis de l'expérience - ou VAE), remettent en cause les conventions établies quarante ans plus tôt, conventions qui n'avaient pas été négociées avec les intéressés (les représentants du monde professionnel) et qui déniaient l'expérience parce que celle-ci ne

${ }^{13}$ Loi n 89.486 du 10 juin 1989. pouvait, à l'époque, donner lieu à une définition générale. Les déplacements effectués en matière de relations entre l'éducation et le travail, ou la formation et l'emploi, sont à l'origine de l'invasion de la notion de compétence dans tout l'espace public.

Le modèle pédagogique des compétences représente peut-être l'exemple le plus éloquent de ce changement de perspective pédagogique qui a progressivement pénétré l'institution scolaire depuis la publication de la «Charte des programmes » en 1992 (publiée au Journal Officiel du 6 février 1992). Quelles que soient les formes prises par ces changements dans tel ou tel segment de l'appareil éducatif, elles s'accompagnent toutes de la mise en place de dispositifs techniques, de procédures de catégorisations, de nomenclatures et de classements construits sur le même modèle, à partir d'un découpage entre le domaine des savoirs et des savoir-faire et de pratiques d'évaluation leur correspondant. Ces pratiques s'étendent à l'Université par la récente mise en place du LMD (qui désigne les trois étapes du cursus universitaire, licence, master et doctorat) en vue de faciliter la circulation des étudiants en Europe, de permettre ainsi une comparaison et d'établir des équivalences dans la perspective des ECTS (European Credits Transfert System $)^{14}$. Cette réforme de l'offre de formation dans l'enseignement supérieur emprunte et consacre ce fondement de la doctrine de la formation permanente : un parcours individualisé, composé de modules qui s'agencent au gré du choix des individus (ou des circonstances) et qui sont validés en unités sommables. La standardisation de ces formations pédagogiques pour toutes les disciplines et sur tous les territoires (comme si l'offre y était équivalente) présuppose, en face, un acteur rationnel, doté d'une conscience calculatrice, capable d'adopter une stratégie appropriée à la situation dans laquelle il se trouve. Discutable lorsqu'il s'applique à un individu engagé dans une vie professionnelle et usager de la formation permanente, ce raisonnement devient totalement abstrait appliqué aux étudiants commençant des études supérieures. La standardisation et la simplification de ce type d'organisation occulte ses orientations instrumentales qui s'imposent avec plus

${ }^{14}$ Le lecteur intéressé par cette question se reportera à l'analyse de cette réforme faite par Annie Vinokur dans Formation Emploi (2002). 
ou moins de force selon les ressources économiques et culturelles de l'individu.

Contrairement à une tradition remontant au XIX ${ }^{\mathrm{e}}$ siècle, où les réformes solaires étaient l'objet d'examens et de disputes politiques, les modalités de décision et de mise en application de ces changements s'accomplissent par des procédures sans débat préalable. L'analyse de cet ensemble de transformations en cours $\mathrm{m}$ 'a conduit à poser cette hypothèse : l'éducation et la formation ont été construites comme des instruments et des piliers d'un changement d'ordre social d'envergure mais d'orientation différente. L'éducation a été pensée comme le vecteur d'un futur à faire advenir par le registre politique tandis que la formation est, depuis ses débuts, plus fortement arrimée au registre économique. Le glissement sémantique de la notion d'éducation à celle de formation recouvre, entre autres choses, la différence de statut occupé par ces deux domaines d'activités qui s'adressent à des publics différents définis par leur position dans le cycle de la vie. L'éducation ainsi que le lieu où elle s'accomplit, l'École, se sont constituées, en France, au terme de conflits politiques qui lui ont imprimé des marques de neutralité politique et de laïcité, lesquelles sont toujours objet de mobilisations sociales pour défendre des points de vue contre d'autres. La formation est une activité où se réalise l'accord, où la coopération entre acteurs s'opère sans heurts importants. Cet accord s'est construit, partiellement, dans le malentendu mais aussi dans une acception de l'intérêt général associé à la définition d'une économie compétitive, d'une modernisation de la société, de la mise en place d'institutions de participation et de dialogue social dans un contexte de recherche de réduction des conflits sociaux et politiques. À la différence de l'École qui a été une affaire d'État, la formation a progressivement été élaborée comme l'affaire des «partenaires sociaux», mais sous l'impulsion et le contrôle constants de l'État.

Promue comme un bien collectif et un instrument de réformes, la formation permanente s'avère avoir été un principe actif de la rationalisation, et de la modernisation des entreprises recherchée par les décideurs économiques et politiques. Mais, aujourd'hui, elle ne peut être dissociée de son revers : les changements dans les rapports salariaux se traduisent par une intensification de la vulnérabilité des salariés sur le marché du travail où la sélection entre les différentes catégories de population, jeunes ou plus âgés, hommes ou femmes, ouvriers ou employés ou cadres, s'effectue au nom de la formation sous ses différentes formes, le diplôme, les certifications professionnelles, les validations de compétences, etc. Non seulement la formation continue s'avère plus inégalitaire que la formation initiale ${ }^{15}$ mais elle reste étrangère aux schémas de perception de la fraction des salariés les plus démunis sur le marché de l'emploi.

\section{De la formation à la certification}

La rationalisation ainsi recherchée, d'ordre éminemment instrumentale, fondée sur l'idée d'évaluation des acquisitions dans des situations données au moyen de la notion d' " être capable de », grâce à des outils qui permettent de les vérifier, a trouvé une application extensive dans les entreprises qui s'emploient à délivrer leurs propres certifications. Les nouvelles formes de certifications, instituées depuis le milieu des années 90, appelées certificats de qualifications professionnelles (CQP), délivrées par les Commissions paritaires de l'emploi, sont construites à partir de cette méthode des référentiels. Ici, comme là, il s'agit de valider les capacités d'accomplir des tâches données dans des situations données.

Les changements en cours, en matière de certification, proviennent, pour l'essentiel, d'initiatives du monde de la formation. La loi de modernisation sociale (2002) qui entérine de nouveaux modes de certification représente, elle aussi, un véritable basculement des institutions et des pratiques instaurées depuis plus d'un demi siècle: en termes de lieux, d'instances, d'acteurs, de pratiques telles que celles de validation des acquis de l'expérience. Ces changements font aujourd'hui débat et sont l'objet d'études qui interrogent les dispositifs et procédures mis en place à cet effet: un Comité national des certifications professionnelles (CNCR) et un Référentiel national des certifications professionnelles (RNCP). Ils prennent place, et trouvent peut-être leur sens, dans la formation de l'espace politique

${ }^{15}$ Le CEREQ fournit là aussi des références statistiques essentielles qui permettent de dresser un tableau de la persistance des inégalités d'accès à la formation continue selon les catégories socioprofessionnelles et selon d'autres caractéristiques, d'entreprises notamment. 
européen où a été créé un «cadre européen des certifications ». Les premiers travaux impulsés par Fabienne Maillard et José Rose (2007) tentent de saisir les possibles contenus dans ces glissements du diplôme, dont l'État détenait le monopole de la délivrance et qui était au fondement des classifications professionnelles entérinées par les conventions collectives de branche, vers un régime généralisé de certifications partielles institué parallèlement à une gestion du travail salarié en termes de compétences, qui postule que tout salarié doit constamment faire la preuve de sa capacité à occuper un poste donné. La durée manque pour observer au plus près des situations concrètes comment se réalisent ces innovations et comment elles modifient les relations salariales. La dissociation tendancielle ainsi introduite, soulignée par tous, entre formation et certification, est-elle de nature à rendre possible la reconnaissance des compétences des salariés dans les classements établis par les entreprises et les salaires qui leur sont attachés où, au contraire, à justifier le déni aujourd'hui exercé ? L'adhésion des organisations syndicales à l'instauration de ce régime des certifications nous conduit naturellement à examiner les relations sociales du travail dans lesquelles celle-ci s'effectue.

\section{Retrait de l'État} et dialogue social entre partenaires

Le sens du terme «dialogue social» varie avec le statut de ses utilisateurs. Il est aujourd'hui intégré dans le vocabulaire du monde professionnel et politique pour nommer un objectif recherché qui n'est le plus souvent désigné qu'en creux : instituer des relations entre des groupes aux intérêts divergents et éviter des oppositions susceptibles de détourner les « forces vives » d'actions concertées dites être dirigées vers un intérêt commun. Les formes et procédures utilisées à cet effet évitent d'expliciter un contenu par trop ambivalent. Par maints aspects, le dialogue social peut être considéré comme une catégorie politique liée aux changements de modes de gouvernement des États, mais aussi des organisations professionnelles, pour signifier la substitution du contrat à la loi comme principe de fonctionnement et de légitimité. En définitive, cette notion prend sens dans une configuration de mots qui lui sont constam- ment associés dans les discours et les pratiques: participation, concertation, partenariat, paritarisme, contractualisation.

La formation a été considérée, par ses promoteurs, et Jacques Delors notamment, comme un vecteur permettant de transformer en profondeur le régime des relations de travail en instituant, entre autres choses, le paritarisme dans la gestion de son financement. La loi du 4 mai 2004, qui associe explicitement deux phénomènes jusqu'alors perçus comme sans liens apparents, la formation tout au long de la vie et l'instauration du dialogue social dans les entreprises, s'inscrit dans un processus de longue durée, dont la portée n'est pas encore mesurable. Mais, alors que les salariés sont restés jusqu'à peu de temps dans une attitude relativement distante à l'égard de la formation, leurs organisations syndicales l'ont désormais intégrée dans leurs doctrines et leurs stratégies sous des modes différents. On citera l'exemple des deux confédérations dont les points de vue sur la formation divergent de longe date : la CGT (Confédération générale du travail) et la CFDT (Confédération française démocratique du travail). La CGT est passée d'une défiance (durant les années 1950-60) à une adhésion sceptique après 1970, en incluant l'idée de formation dans une perspective générale de lutte pour l'emploi et contre les inégalités pour, ces toutes dernières années, l'intégrer dans un programme prospectif définissant «Un nouveau statut de travail salarié ». Elle propose d'instaurer une "sécurité sociale professionnelle» qui substituerait à une vision réparatrice de l'indemnisation du chômage et du droit sur les licenciements, un droit de l'individu tout au long de sa vie qui le libère de sa dépendance au devenir et à la gestion de telle ou telle entreprise. Arrimée à l'emploi, depuis 1971, la formation est maintenant considérée comme partie intégrante d'un ensemble de droits collectifs interdépendants à défendre face au chômage mais aussi face aux tendances générales à l'individualisation qui travaillent notre société. Héritière des mouvements catholiques, la CFDT a toujours placé la formation dans sa matrice d'action. Réalisée dans l'école ou sur les lieux de travail, celle-ci est toujours parée, au moins potentiellement, de vertus émancipatrices. L'association entre les trois termes, formation professionnelle continue, 
dialogue social et politique contractuelle, constamment faite par les dirigeants de ce syndicat, exprime d'une manière condensée sa doctrine en matière d'action collective et ses préférences pour un régime social où la négociation et la coopération prévalent sur la contestation et la projection d'un ordre social radicalement différent. ${ }^{16}$

Plus généralement, le domaine de la formation s'avère être un exemple du mode de gouvernement par contrat, où la région ${ }^{17}$ (ou plus spécifiquement le conseil régional) devient l'acteur central qui tisse des contrats d'objectifs, avec les branches professionnelles et autres groupes d'intérêts organisés sur le territoire, en matière de formation. Par la mise en place de PRDFPJ (Plans régionaux de développement de la formation professionnelle des jeunes) qui incluent l'enseignement professionnel, l'apprentissage, les actions de formation destinées aux 1625 ans déclarés sans qualification à la sortie de l'appareil scolaire, les contrats d'insertion des jeunes, l'État s'est, en effet, attaché à instaurer des procédures de constitution et de mise en relation des différents groupes d'intérêts présents dans l'espace régional. Mais la loi ne fait que poser le cadre des interactions entre acteurs et institutions concernés par la politique de formation des jeunes : instances étatiques régionales, collectivités locales, organisations professionnelles patronales et salariés, entreprises, etc. Le caractère procédural de la loi autorise une grande variété d'arrangements locaux (Lascourmes, 2003). Dans le domaine de la formation professionnelle des salariés actifs, la loi est encore moins contraignante puisqu'elle laisse l'initiative aux «partenaires sociaux » et ne fait que poser des bornes à celle-ci.

Dans une conjoncture marquée par le parallélisme, ou l'interdépendance, de changements aussi mani-

\footnotetext{
${ }^{16}$ Plus globalement, la CFDT fait sienne cette vision diffusée par les organismes internationaux et européens (parmi lesquels les textes de la Commission européenne) selon laquelle la « formation tout au long de la vie » engloberait la formation initiale jusqu'à maintenant majoritairement accomplie dans l'école en France. Colloque organisé par ce syndicat, « La formation tout au long de la vie, levier de la réforme de l'école », Syndicalisme Hebdo, $2004, n^{\circ} 3000$

${ }^{17}$ La loi quinquennale du 20 décembre 1993, relative au travail, à l'emploi et à la formation professionnelle, transfère la formation professionnelle des jeunes aux conseils régionaux, compétence étendue aux adultes non salariés depuis 2002.
}

festes, il importerait de pouvoir observer aussi bien la mise en application de la VAE que celle des systèmes de certification et de leurs effets en matière de reconnaissance dans les parcours professionnels notamment. Ces nouveaux besoins en connaissance, apparus corrélativement à ces transformations institutionnelles, seront-ils inscrits dans le programme de recherche annoncé au terme du rapport, de mon point de vue mal nommé18, La certification, nouvel instrument de la relation formation-emploi (Teissier, Rose, 2006) ? Tout pousse à ouvrir grand l'angle de vue pour rendre compte des différentes dimensions revêtues par l'instauration d'une « Formation tout au long de la vie » associée à un régime de " dialogue social ». Le CEREQ, par les outils d'observation et d'analyse dont il s'est doté dans le temps et par l'expertise qu'il a acquise dans ce domaine, se trouve tout désigné pour remplir cette mission qui n'est, en définitive, qu'une forme renouvelée de celles accomplies jusqu'à maintenant.

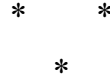

L'inflation actuelle des discours sur la formation tout au long de la vie n'est pas sans rappeler celle tenue sur l'éducation des adultes au cours des deux décennies qui précèdent la loi de 1971 en France. Les analyses qui en ont été faites montrent que ces discours reposaient, à quelques variantes près, sur les mêmes thèmes que ceux célébrés aujourd'hui par la Commission européenne (Forquin, 2002). Mais, alors que les acteurs qui plaidaient, dans les années 1960-1975, pour l'avènement d'une ère de l'Éducation permanente se rassemblaient autour de l'UNESCO et préconisaient des politiques d'éducation et de formation répondant à des demandes collectives et mettant en œuvre des programmes et des dispositifs éducationnels fortement institutionnalisés, les politiques actuelles sont davantage orientées vers la demande et la responsabilité individuelle des apprenants. On ne saurait donc sous-estimer les changements qui se produisent au niveau des

\footnotetext{
${ }^{18}$ Ce titre inscrit l'analyse des phénomènes observés dans une perspective désignée par cette locution, dont j'ai montré plus haut les effets pervers, et qui, de surcroît, occulte le détachement de la certification d'une formation préalable.
} 
programmes européens et des législations nationales encadrant les pratiques de formations, parmi lesquels: l'individualisation, le déplacement du temps de travail vers le temps de vie personnelle. Corrélativement, le rôle des pouvoirs publics dans l'organisation, la gestion et le financement du système d'éducation et de formation perd de l'importance, au profit de modèles de partenariat et de partage de responsabilités. Toutes ces transformations impliquent un déplacement de l'autorité de l'État vers une diversité d'acteurs de la vie économique et politique. Elles sous-tendent l'inscription de la notion de dialogue social, et des mécanismes institutionnels producteurs de références communautaires que cette notion désigne, dans l'ensemble de l'espace européen, dont la paternité reviendrait, pour une large part, à J. Delors (Didry et Mias, 2005).

Le caractère polymorphe de cette notion favorise la plasticité de son usage dans le cadre national comme européen, mais son introduction dans le projet de Traité constitutionnel à côté du principe de démocratie représentative et de celui de démocratie participative indique bien les visées de ses promoteurs: en faire un principe de fonctionnement de la vie sociale et politique de l'Union. ${ }^{19}$
Ainsi appréhendée, la formation apparaît comme une notion carrefour où se croisent diverses composantes des politiques libérales promues à l'échelle européenne. Ces dernières reposent, dit K. Rubenson (ibid, p. 226), sur une conception de la vie représentée comme « une entreprise de soi » qui exige que chacun agisse en vue de créer et conserver son capital humain. Investir dans une formation relève alors de la responsabilité individuelle. Cette idéologie exclut l'intervention de l'État et des institutions publiques qui œuvraient, dans une période antérieure, à corriger les inégalités sociales dans ce domaine comme dans d'autres.

Un espace de recherche s'ouvre avec ces transformations où la formation est toujours pensée comme un vecteur efficient, et où le CEREQ devrait, de par son histoire, être en mesure de définir des programmes de recherche à grande échelle et de style pluridisciplinaire.

${ }^{19}$ TitreVI, article I-47, Principe de la démocratie participative, al. 2 « Les institutions entretiennent un dialogue ouvert, transparent et régulier avec les associations représentatives et la société civile »; et article I-48, Les partenaires sociaux et le dialogue social autonome, al. 1 et 2 .

\section{Bibliographie}

Bertrand H. (2001), «Introduction», Formation Emploi, $\mathrm{n}^{\circ}$ spécial «Trente ans d'analyses », 76, pp. 7-12.

Briand J.-P., Chapoulie J.-M. (1993), «L'institution scolaire et la scolarisation: une perspective d'ensemble », Revue française de sociologie, 34, 4, pp. 3-42.

Brucy G., Caillaud P., Quenson E., Tanguy L. (2007), Former pour réformer. Retour sur la formation permanente 1945-2004, Paris, La Découverte.
Casella Ph. (1999), «La mobilisation par l'administration d'un monde professionnel de la formation », Sociétés contemporaines, numéro spécial (35), pp. 95-115.

CEREQ (1973), «Les activités du CEREQ en $1973 »$, Note d'information, $n^{\circ} 11$.

CEREQ (1978), « Ce que l'on sait aujourd'hui du travail dans ses relations avec la formation: les acquis scientifiques après sept années d'activités du CEREQ », Note d'information $\mathrm{n}^{\circ} 52$, p. 9 
Didry C., Mias A. (2005), Le moment Delors. Les syndicats au cour de l'Europe sociale, Peter Lang, Bern.

D'Iribarne A. (2001), « Trente ans de CEREQ - Des qualifications aux compétences: chronique d'un oubli accepté ?», Formation Emploi, n ${ }^{\circ}$ spécial « Trente ans d'analyses », 76, pp. 71-99.

Donnadieu J.-L. (1999), D'hommes à hommes, itinéraire d'un DRH, L'Harmattan, Paris.

Darhendorf (cité par Beate Krais, 1995), «Trente ans de sociologie de l'éducation: problématiques et controverses ", in A. Jobert, C. Marry, L. Tanguy, Éducation et Travail en Grande-Bretagne, Allemagne et Italie, Armand Colin, pp. 48-61.

Dupray A., Guitton C., Monchatre S. (dir.), (2003), Réfléchir la compétence, approches sociologiques, juridiques, économiques d'une pratique gestionnaire, Octarès, Toulouse.

Forquin J.-C. (2002), Les composantes doctrinales de l'idée d'éducation permanente, analyse thématique d'un corpus international (UNESCO), L'Harmattan, Paris, pp. 7-45.

Giret J.-F., Lopez A., Rose J. (dir.), (2005) Des formations pour quels emplois?, Paris, La Découverte.

Larsson S. (2001), « Les cercles d'études et la démocratie en Suède, Éducation permanente, 2001, n ${ }^{\circ}$ 149, pp. 231-256.

Lascoumes P (2003), « Gouverner par les instruments, ou comment instrumenter l'action publique?» in Lagroye J. (dir.), La politisation, Belin, Paris, pp. 387-401.

Maillard F. (2005), Pour un regard sociologique sur la formation et les diplômes professionnels, Habilita- tion à diriger des recherches, université d'Amiens, $2^{\mathrm{e}}$ partie, «Les diplômes professionnels, construction et enjeux ».

Maillard F., Rose J. (2007), Les diplômes de l'Éducation nationale dans l'univers des certifications professionnelles. Nouvelles normes et nouveaux enjeux, CEREQ, Mars.

Méhaut Ph. (2001), "Gouverner les systèmes de formation professionnelle: planification, marché, coordination?», Formation Emploi, n spécial 76, pp. 225-235.

Ropé F. , Tanguy L. (dir.) (2004), Savoirs et compétences, de l'usage de ces notions dans l'école et l'entreprise, Paris, l'Harmattan.

Rubenson K. (2001), «L'éducation des adultes en Suède de 1967 à 2001. De l'éducation permanente à l'apprentissage tout au long de la vie », Éducation permanente, $\mathrm{n}^{\circ} 149$, pp. 211-230.

Tanguy L. (1991), Quelle formation pour les ouvriers et les employés en France?, rapport de mission Secrétariat d'État à l'Enseignement Technique, Paris, La Documentation française.

Teissier J., Rose J. 2006 (Éds.), «La certification, nouvel instrument de la relation formation emploi. Un enjeu français et européen », CEREQ, Relief, $\mathrm{n}^{\circ} 16$.

Vatier R. (1958), La formation et les institutions de l'entreprise, Journée d'études de l'IFPC, archives des GARF.

Vinokur A. (2002) «Enseignement supérieur: un changement sans réforme?», Formation Emploi, $\mathrm{n}^{\circ} 79$, juillet-septembre, pp. 19-3. 


\title{
Résumé
}

\section{La recherche de liens entre la formation et l'emploi : une institution et sa revue - Un point de vue -}

\author{
Lucie Tanguy
}

Un bref retour sur la création du CEREQ, des missions qui lui sont conférées et sur la revue née quelques années plus tard, introduit la rétrospective qui suit sur la constitution d'un domaine de recherche désigné par la locution «la relation formation/emploi » que l'auteur discute. Promue comme un bien collectif et un instrument de réformes, la formation permanente s'avère avoir été, durant les décennies 1970 et 1980, un principe actif de la modernisation des entreprises recherchée par les décideurs économiques et politiques. Les quinze dernières années sont également marquées par des changements d'ampleur équivalente, et la formation est toujours convoquée comme instrument nécessaire à leur accomplissement dans l'emploi et le travail, mais, présentée comme un droit individuel, elle s'avère plutôt être une obligation pour garder un emploi. Associée à l'instauration d'un régime de "dialogue social », la formation apparaît aussi comme une catégorie politique.

\section{Mots clés}

Relation formation-emploi, formation professionnelle, représentation de la formation, négociation collective Journal of Economic Literature: J 52 - Dispute Resolution: Strikes, Arbitration, and Mediation - Collective Bargainin ; J 53 - Labor - Management Relations - Industrial Jurisprudenc ; M 53 - Training 


\section{Cahiers du Genre- n $44 / 2008$}

- Dossier

Gender mainstreaming:

de l'égalité des sexes à la diversité ?

Coordonné par

Sandrine Dauphin et Réjane Sénac-Slawinski

$\square$ Sandrine Dauphin et Réjane Sénac-Slawinski

Gender mainstreaming : analyse des enjeux d'un 'concept-méthode' (Introduction)

$\square$ Geneviève Fraisse

Le gender mainstreaming, vrai en théorie, faux en pratique?

(Interview par Sandrine Dauphin et Réjane Sénac-Slawinski)

- Réjane Sénac-Slawinski

Du gender mainstreaming au paritarisme : genèse d'un concept controversé

- Maria Stratigaki

La politique du recul. De l'intégration de l'égalité 'des sexes' à l'intégration de l'égalité 'pour tous'

$\square$ Judith Squires

Diversity mainstreaming : dépasser les approches technocratiques et d'addition des inégalités

$\square$ Alison E. Woodward

Est-il trop tard pour une approche intégrée de l'égalité ? Le point sur la situation à Bruxelles

$\square$ Diane Sainsbury et Christina Bergqvist

Gender mainstreaming 'à la suédoise'

๑ Sandrine Dauphin

Promotion de l'égalité des sexes en France : continuité et rupture

- Marie-Thérèse Letablier et Gwenaëlle Perrier

La mise en œuvre du gender mainstreaming dans les politiques locales de l'emploi. L'exemple du Fonds social européen en Île-de-France

\section{- Hors-champ}

a Sabine Masson

Histoire, rapports sociaux et mouvements des femmes indiennes au Chiapas (Mexique). Sur I'usage de l'histoire dans la recherche féministe postcoloniale

\section{- Lecture d'une œuvre}

Irène Jami

Judith Butler, théoricienne du genre

Notes de lecture

Vente au numéro : à la librairie L'Harmattan et dans les librairies spécialisées Les abonnements sont annuels et partent du premier numéro de l'année en cours : France : $45 €-$ Étranger : $50 €$ frais d'envoi compris Veuillez adresser votre commande

aux éditions L'Harmattan - 5-7 rue de l'École Polytechnique 75005 Paris

Tél. 0140467920 - Mél diffusion-harmattan@wanadoo.fr

Pour tout autre renseignement sur la revue, s'adresser au secrétariat de rédaction :

Danièle Senotier tél. 0140251165 - mél. daniele.senotier@gtm.cnrs.fr http://cahiers_du_genre.iresco.fr/ 